\title{
Oilless and frictionless compressor with double-sided rotationally oscillating piston
}

\author{
W. CHOMCZYK ${ }^{1}$, W. OSTAPSKI ${ }^{2 *}$ J. PIOTROWSKI ${ }^{2}$, and T. TATAJ ${ }^{2}$ \\ ${ }^{1}$ Bialystok University of Technology, 45D Wiejska St., 15-351 Bialystok, Poland \\ ${ }^{2}$ Institute of Machine Design Fundamentals, Warsaw University of Technology, 84 Narbutta St., 02-524 Warszawa, Poland
}

\begin{abstract}
The contents are concerned with a concept of an oilless and frictionless compressor operating without dead movement and having functional parameters superior to compressors of renowned manufacturers. The concept is based on patents No. 68220, 83060, P377851 by W. Chomczyk and patents P394072, P385733 by W. Chomczyk, W. Ostapski and J. Piotrowski. The proposed design of an oilless compressor ensures volumetric efficiency higher than that encountered in conventional compressors. It also generates pure compressed medium without necessity of filtering from oil particles, which is especially desired in food, pharmaceutical, medical, fragrance, chemical and automation industry.
\end{abstract}

Key words: oilless and frictionless compressor, double-sided rotationally oscillating piston.

\section{Introduction}

The principle of working of an oscillatory compressor is given on the scheme in Fig. 1. In cylindrical case (1), there are two alternately placed partition walls (2) equipped with four outlet valves (3). Concentrically mounted shaft (4) has two vanes (5) being an equivalent to the piston. The vanes can rotate in the free space between the partition walls by an angular displacement $\phi_{R}$. On the cylindrical case, there are two inlet valves (6) installed. Motion of the shaft compresses the gas in chamber (7) and at the same time decompresses it in chamber (8). At the moment when vanes (5) uncover valve seats (6), the gas suddenly inflows to chamber (8) due to a considerable difference in the pressure. Dead point (1) of rotating shaft is at the distance $\delta$ from the barriers. After reaching it, the shaft changes direction of its rotary motion, owing to which in chamber (8) there compression starts and in (7) expansion of the medium.

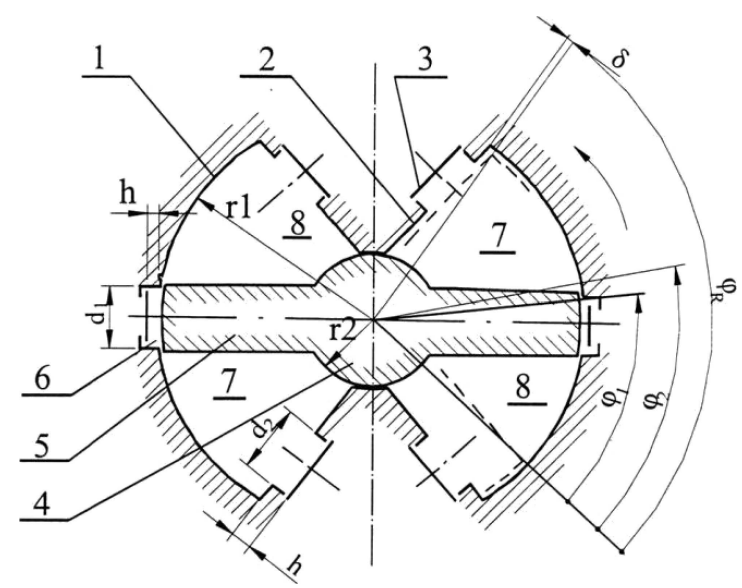

Fig. 1. Scheme of the oscillatory compressor

\section{Working cycles of the oscillatory compressor}

Due to the displacement of the inlet valve at the half of the suction stroke and because of the clearance volume above the inlet valve seat, the ideological cycle of working of the considered compressor slightly differs from a conventional scheme incorporated to piston compressors. Beginning from dead point (1), in which the outlet valve closes, there starts decompression with a polytropic exponent $m_{1}$. It lasts till vane (5) uncovers by its rear edge the seat of the inlet valve (6), i.e. at the angular displacement $\varphi_{2}$, slightly greater than the angle $\varphi_{R}$. In Fig. 1, the abscissa depicts the displacement volume. Accordingly, point (2) of the opening of the inlet valve corresponds to volume of the decompression chamber $\left(V_{1}+V_{\varphi 2}\right)$. At this place, the compressed gas over the inlet valve inflows to suction chamber (8), increasing pressure by $\Delta p_{2}^{\prime}$. Starting from point (2'), suction chamber (8) is dynamically filled with the gas, which ends at dead point (3). The loss of kinetic energy of the gas makes the chamber pressure near point (3) practically the same as the pressure in the suction collector.

Basically, compression goes along a polytropic curve with the exponent $m_{1}$. However, after reaching the angular displacement $\varphi_{1}$, the space over the inlet valve $\Delta V_{3}$ becomes closed from pressure chamber (7) and the compression polytropic curve shifts from point $3^{\prime}$ to $3^{\prime \prime}$. Point 4 corresponds to the opening of the outlet valve, which - as in conventional compressors - takes place at a higher pressure than that in the pressure collector $p_{t}$. Arriving to dead point (1), the velocity of the rotary piston and the resistance of flow through valve (3) approach zero, hence the pressure surplus $\Delta p_{t}$ over the pressure in the outlet collector corresponds to the force produced by the spring supporting the outlet valve. As can be seen here, the thermodynamic cycle of the presented compressor differs from cycles of typical compressors with reciprocating pistons.

*e-mail: wos@simr.pw.edu.pl 
W. Chomczyk et al.

The beginning of decompression can be described by the state of the gas determined by known parameters and conditions: the clearance volume ensuing from the design, pressure $p_{t}$ equal to that in the outlet collector and the surplus $\Delta p_{t}$, which depends on the force closing the check valve. It amounts to:

$$
\Delta p_{t}=\frac{4 \delta_{2}}{\pi d_{2}^{2}}
$$

where $\delta_{2}$ is the pressure from the spring that pushes the plate of the valve. By denoting: $p_{1}=p_{t}+\Delta p_{t}$ one can find the pressure at point (2) from the polytropic equation. The closed in chamber (8) gas undergoes quite intensive heat exchange with the chamber walls during decompression. Assuming, as it is in piston compressors, that at the ratio $p_{t} / p_{4} \geq 4$ the empirical polytropic exponent of decompression equals $m_{1}=1.2$, one obtains for $V_{2}=V_{1}+V_{\varphi 2}$ :

$$
p_{2}=\left(\frac{V_{1}}{V_{2}}\right)^{1.2} p_{1} .
$$

At this moment, the space covered by vane (5) over valve (6) exposes. Its volume amounts $\Delta V_{3}=h \pi d_{1}^{2} / 4$. The pressure $p_{3}^{\prime}$ is not yet known, however due to its poor influence on the increment $\Delta p_{2}$ it is assumed that $p_{3}^{\prime}=2.9 p_{n}$. For isotropic mixing $p_{2} V_{2}+p_{3}^{\prime} \Delta V_{3}=p_{2}^{\prime} V_{2}$, we get:

$$
\Delta p_{2}=2.9 p_{n} \frac{\Delta V_{3}}{V_{2}} \text {. }
$$

Thus the state of the gas at point $2^{\prime}$, being the start of dynamic filling-in, has been determined.

Let, at any arbitrary point of filling-in (Fig. 2), the volume of the suction chamber be $V_{1}+V_{\varphi 2}+V$, where $V \approx$ $0.5 V_{R} \sin \omega t$. The corresponding to that point pressure differs from pressure in the suction pipe by $\Delta p=p_{n}-p$. From the law of momentum conservation, for the cross-section of the valve gap $F$ and the volume $V$ enclosed in it, yields:

$$
F \Delta p \mathrm{~d} t=V_{s} \rho \mathrm{d} v, \quad \Delta p \mathrm{~d} t=L \rho \mathrm{d} v,
$$

where $\rho$ is the gas density $\left[\mathrm{kg} / \mathrm{m}^{3}\right], v-$ gas velocity in the gap of the suction valve $[\mathrm{m} / \mathrm{s}], L-$ length of the acceleration path in the gap equal to $0.0001[\mathrm{~m}]$.

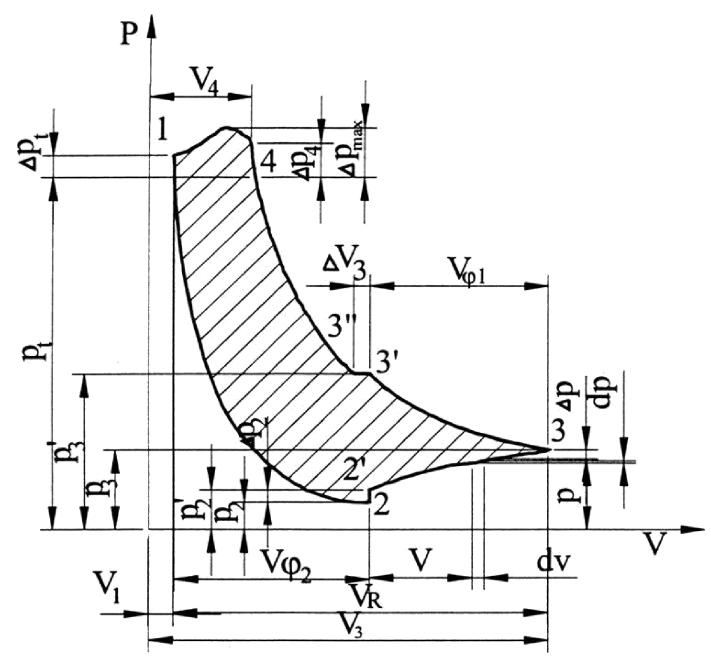

Fig. 2. Working cycle of the oscillatory compressor
To the drop of pressure by, there corresponds the flow velocity:

$$
v=\sqrt{2 \frac{\chi}{\chi-1} \cdot \frac{p}{\rho}\left[1-\left(\frac{p_{n}}{p}\right)^{\frac{\chi-1}{\chi}}\right]} .
$$

For simplification of the calculus, Bernoulli's relationship $\Delta p=\rho\left(v^{2} / 2\right) / \alpha$ with variable density $\rho$ has been assumed. Such an approximation gives some insight into dynamics of the filling-in, especially when the pressure $\Delta p$ is less than the critical one. However, at low values of the outlet pressure, in point $2^{\prime}$ at the beginning of filling-in, the pressure differs from the external one by a value greater than the critical, owing to which the outlet velocity is limited by the speed of sound. This difference is so fast compensated that it should not considerably affect the ordinate of point 3 at the end of the filling-in. Too detailed calculations would be unjustified here because of many factors that cannot be univocally presented in an analytical manner such as disturbances generated by structural features of the valves, vorticity in the suction chamber, etc. Therefore, much more reliable value of pressure in the point of interest (3) could be found from the indicator diagram.

From Bernoulli's equation, we have: $v=\sqrt{2 \alpha \Delta p / p}$ and $d v=\sqrt{\alpha /(2 \Delta p \rho)}$. Having substituted these expressions into Eq. (4), for the coefficient of loss $\alpha=0.45$, one obtains:

$$
\begin{gathered}
10 \Delta p \mathrm{~d} t=\frac{\alpha \mathrm{d} p}{V} \cong \frac{\alpha \mathrm{d} p \sqrt{\rho}}{\sqrt{2 \alpha \Delta p}}, \\
\mathrm{~d} t=0.0474 \sqrt{\frac{\rho}{\Delta p}} \mathrm{~d} p .
\end{gathered}
$$

It is assumed that during the process of filling along states $2^{\prime}-3$, the pressure variation is isothermic, then the corresponding density $\rho$ would be smaller than the real value for a process close to adiabatic and, consequently, the final result of the filling-in would be more disadvantageous from the real one. This enables introducing a simpler relationship for $\rho$, which with respect to $\left[1 \mathrm{~cm}^{3}\right]$ and the gas constant for air $R \approx 29.3 \times 10^{2}$ at $T=300 \mathrm{~K}$ equals $\rho^{1 / 2}=$ const $\cdot p^{1 / 2} \cdot 294 \times 10^{-4}$.

In order to replace in (5) the expression dt with known physical parameters, one introduces a differential volume brought about by changes in the volume $V \approx 0.5 V_{R} \sin \omega t$ :

$$
\mathrm{d} V \approx 0.5 V_{R} \omega \cos \omega t \cdot \mathrm{d} . t
$$

For an oscillatory character of the piston motion, we have $\omega=0.5 \pi\left(V / V_{R}\right)$. By denoting: $\omega=0.5 \pi V_{R}=K$, and additionally $\omega t=K V$, that is:

$$
\mathrm{d} v=0.5 V_{R} \omega \cos (K V) \cdot \mathrm{d} t .
$$

In the assumed units of measure, the pressure is expressed in $\left[\mathrm{kG} / \mathrm{cm}^{2}\right]$, thus one can accept the pressure of filling-in $p_{n} \approx 1$, and the resulting difference $\Delta p_{n}=1-p$. After substituting the above mentioned values into equation (5), the following formula is obtained:

$$
\frac{\mathrm{d} V}{\cos (K V)}=\sqrt{\frac{p}{(1-p)^{3}}} \mathrm{~d} p .
$$


Having substituted $K V=X$ and integrated, from the expression $\mathrm{d} V / \cos (K V)$ one finds:

$$
\begin{gathered}
\frac{1}{K} \int \frac{\mathrm{d} x}{\cos X}=\frac{1}{K} \ln \left[\tan \left(\frac{\pi}{4}+\frac{X}{2}\right)\right] \\
=\frac{2 V_{R}}{\pi} \ln \left[\tan \left(\frac{\pi}{4}\left(1+\frac{V}{V_{R}}\right)\right)\right] .
\end{gathered}
$$

The integral $I=\int \sqrt{\frac{p}{(1-p)^{3}}} \mathrm{~d} p$ can be determined by making use of the substitution $t=1-p$, which yields $p=1-t^{2}$, $\mathrm{d} p=-2 t \mathrm{~d} t$, and

$$
I=\left(\frac{1+p}{1-p}\right) \sqrt{p(1-p)}+\arcsin \sqrt{1-p} .
$$

By assuming that at the end of the filling-in (point 3 in Fig. 2) $p \rightarrow 1\left[\mathrm{kG} / \mathrm{cm}^{2}\right]$, the above integral tends to:

$$
I=\sqrt{p \frac{3-p}{1-p}} \approx 2 V \sqrt{\frac{p}{1-p}} .
$$

For the boundary condition $V=0$ and $p=p_{2}^{\prime}$, Eqs. (6a) and (6b) give the integration constant:

$$
C=1.6 \times 10^{-7} \omega V_{R} \sqrt{\frac{1}{1-p_{2}^{\prime}}}
$$

as well as the particular solution to (6) in the form:

$$
\begin{gathered}
\frac{1}{K} \int \frac{\mathrm{d} x}{\cos X}=\frac{1}{K} \ln \left[\tan \left(\frac{\pi}{4}+\frac{X}{2}\right)\right] \\
=\frac{2 V_{R}}{\pi} \ln \left[\tan \left(\frac{\pi}{4}\left(1+\frac{V}{V_{R}}\right)\right)\right] \\
=2.53 \times 10^{-6} \omega\left[\frac{1}{\sqrt{1-p}}-\frac{1}{\sqrt{1-p_{2}^{\prime}}}\right]
\end{gathered}
$$

In the point of interest ( 3 ), the volume $V \approx 0.5 V_{R}$ constitutes the right-hand side of equation (7) equal to 0.88. After substituting it to (7), the following expression is obtained:

$$
\frac{1}{\sqrt{1-p}}-\frac{1}{\sqrt{1-p_{2}^{\prime}}}=3.48 \times 10^{5} \frac{1}{\omega} \text {. }
$$

Because in point $2^{\prime}$ one may come across the absolute pressure within $0.28=0.55\left[\mathrm{kG} / \mathrm{cm}^{2}\right]$, the term $\sqrt{1-p_{2}^{\prime}}$ is slightly lower than 1 and can be neglected comparing with the value of the right-hand side of Eq. (8), so that the thus created error would not exceed $1 \%$. Hence, the pressure in point (3) can be expressed with sufficient accuracy by the following formula

$$
\frac{1}{\sqrt{1-p}}=3.48 \times 10^{5} \frac{1}{\omega} .
$$

It issues from the above that within limits of practical rotational speed of the crank driving the compressor, $p_{3}$ is less than the filling-in pressure $p_{n}$ by barely $10^{-3} \div 10^{-6}\left[\mathrm{kG} / \mathrm{cm}^{2}\right]$.

Compressing and forcing-out. Because of irregular shape of the compressor chamber, and thus a bit enlarged ratio between the area of the walls and the chamber volume with respect to the cylindrical shape, an enlarged heat exchange between the compressed medium and chamber walls are expected. A slightly different is also the polytropic curve, with the exponent $m \approx 1.35$ this time, in contrast to highspeed piston compressors, in which an adiabatic process is assumed. This remark concerns the second part of the compression curve from point $3^{\prime \prime}$ to 4 . At the same time, along the path $3-3^{\prime}$, compression is less intensive and temperature growth insignificant. One may assume here an adiabatic process with the exponent $m \approx 1.4$. The pressure in point $3^{\prime}$ results from the volume of pressure chamber (7), which at this place amounts to $V_{1}+V_{R}+V_{\varphi 1}=V_{2}$. Hence, for $p_{3} \approx p_{n}$, this pressure equals:

$$
p_{3}^{\prime}=\left(\frac{V_{1}+V_{R}}{V_{2}}\right)^{1.4} p_{n}
$$

Further process goes from point $3^{\prime \prime}$ displaced by $\Delta V_{3}$ (see Eq. (3)) to point 4 . The pressure at this point is greater than $p_{t}$ by $\Delta p_{4}$. Approximately, one may assume this pressure surplus as an average value along the passage of the forced-out medium. It should behave as indicated by the data pertaining to piston compressors, i.e. $\Delta p_{t} \approx(0.3 \div 0.7) \Delta p_{s r}$. It is further assumed that $\Delta p_{4}=2 \Delta p_{t}$, hence:

$$
p_{4} \approx p_{t}+2 \Delta p_{t}
$$

Because for $V_{\varphi 1}+\Delta V_{3}=V_{\varphi 2}$ we have $V_{3}^{\prime \prime}=V_{1}+V_{\varphi 1}$ and $p_{3}^{\prime \prime}=p_{3}^{\prime}$, the sought volume of the compression chamber is:

$$
V_{4}=\left(V_{1}+V_{\varphi_{1}}\right) \cdot\left(\frac{p_{3}^{\prime}}{p_{4}}\right)^{0.74} .
$$

The work of forcing through along the path 4-1 corresponds to the pressure $p_{t}+\Delta p_{s r} \approx p_{t}+2 \Delta p_{t}$. The peak pressure has a bit greater excess $\Delta p_{\max } \approx \Delta p_{t} / 0.3 \approx 3 \Delta p_{t}$. Replacing, at this place, the pressure curve by empirical values from piston compressors barely affect the total error of the entire working cycle, which in other points, has been already simplified.

\section{Selection of the driving mechanism}

An oscillatory compressor can be driven with an arbitrary way provided that it produces rotary and oscillatory movement of the vaned piston. The simplest to incorporate, due to quite big frequency of working cycles and angular displacement of the vane, is a mechanical drive system, also used in classical piston compressors.

There exist many constructional variants of such drive systems. Two of them, the most promising, have been analysed and compared in this paper. Their structural solution is very similar to the crankshaft system widely used in piston compressors as well as internal combustion and steam engines. Its principle of working illustrates Fig. 3.

The power is transmitted from the shaft through the crank (eccentric) of radius $r$ and the connecting rod of length $L$ to the rocker arm directly and rigidly joined with the vane shaft. The shaft, vanes themselves as well as the rocker arm can be manufactured as a single part or a multi-element assembly. In both cases, the angular displacement of the rocker arm is accompanied with the identical displacement of the shaft and vanes, hence in kinematical considerations, they all are treated as a single element of the entire drive system. 
W. Chomczyk et al.

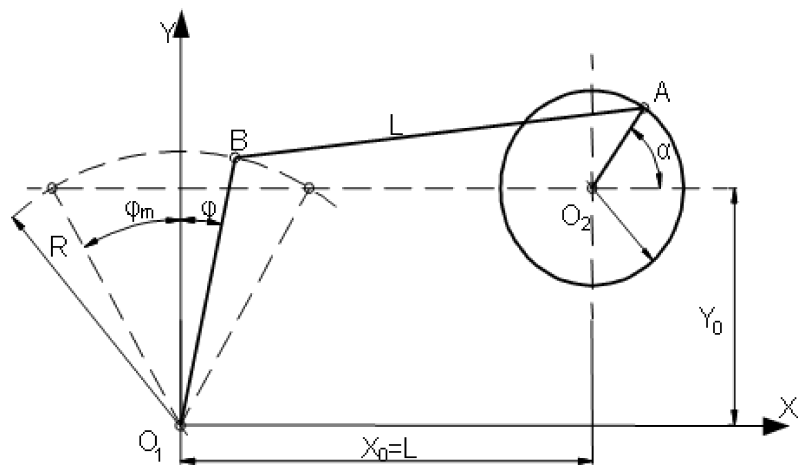

Fig. 3. Scheme of a rock-and-crank linkage (variant I)

There is a following relationship between the maximum displacement $\varphi_{m}$ and the crank radius $R$ :

$$
r=R \sin \varphi_{m} .
$$

In one technologically justified case, when the bilateral extreme oscillations with respect to the axis of symmetry are equal to each other, and yield in those positions the same axial components of the shaft loading, the following condition must be satisfied so that the coordinates of the rotation axis $\mathrm{O}_{2}$ would be:

$$
\left\{\begin{array}{l}
X_{0}=L \\
Y_{0}=R \cos \varphi_{m}
\end{array}\right.
$$

The length of the connecting rod $L$ is also a variable parameter, which - as it is known - gives the smoothest course of acceleration at $L \rightarrow \infty$. For an arbitrary angular position , the crank-pin is placed at position $A$, whereas the pin of the rocker arm is positioned at $B$. According to Fig. 3, the coordinates of both points are:

$$
\begin{gathered}
\left\{\begin{array}{l}
X_{A}=X_{0}+r \cos \alpha \\
Y_{A}=Y_{0}+r \sin \alpha
\end{array}\right. \\
\left\{\begin{array}{l}
X_{B}=R \sin \varphi \\
Y_{B}=R \cos \varphi
\end{array}\right.
\end{gathered}
$$

Their mutual distance is equal to the length of the connecting rod

$$
L^{2}=\left(X_{A}-X_{B}\right)^{2}+\left(Y_{A}-Y_{B}\right)^{2} \text {. }
$$

Or:

$$
\left\{\begin{array}{c}
X_{B}=X_{A}-\sqrt{X_{0}-r^{2} \sin ^{2} \alpha} \\
=X_{0}+r \cos \alpha-\sqrt{X_{0}-r^{2} \sin ^{2} \alpha} \\
Y_{B}=Y_{0} \\
\operatorname{arctg} \frac{X_{B}}{Y_{B}}=\varphi .
\end{array}\right.
$$

By substitute values (15) and (18) into Eq. (19), the following formula is obtained:

$$
\varphi(\alpha)=\operatorname{arctg}\left(\frac{X_{0}+r \cos \alpha-\sqrt{X_{0}-r^{2} \sin ^{2} \alpha}}{Y_{0}}\right) .
$$

For a constant speed of the rotation course, speed and the angular accelerations as a function of the angle of rotation of the shaft can be obtained by calculating the first and second derivative of the function (23) to the variable $\alpha$.

Much faster and precise results can be found by using any modern CAD system. In this paper, CATIA V5 has been employed, which enabled modelling of the compressor parts, defining constraints between them, applying the power drive to any structural node of the system and, finally, simulating the displacements, velocities and accelerations of any point for the given constructional parameters.

The created model (Fig. 4) has been tested in terms of the graphs of interest. Initially, the basic dimensions were assumed: $L=100 \mathrm{~mm}, R=40 \mathrm{~mm}, \varphi_{m}=60^{\circ}$ and a constant rotation speed $n=750 \mathrm{rpm}$.

$$
n=750[\mathrm{rpm}]=4500\left[\frac{\mathrm{deg}}{\mathrm{s}}\right]=\mathrm{const}, \quad \alpha(t)=n t
$$

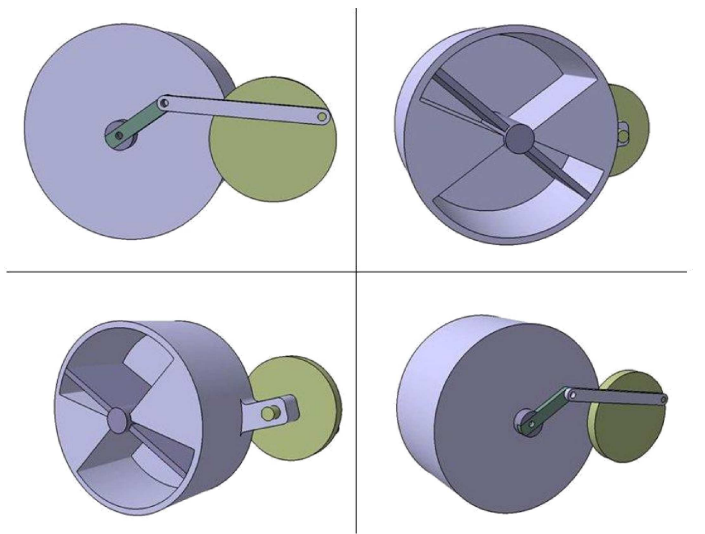

Fig. 4. Model of the compressor drive system (variant I)

In crankshaft systems used in piston compressors, the rotational motion is converted through the connecting rod into linear reciprocation motion of the piston. In the case presented in this paper, the rotational motion of the crank is converted into an oscillatory movement of the rocker arm with the curvature radius $R$ greater than the crank radius $r$ (Eq. (13)). Such kind of motion introduces some irregularities to the drive system, especially vivid in the diagram of angular velocity $\omega(\alpha)$ and acceleration $\gamma(\alpha)$ - see Figs. 5 and 6.

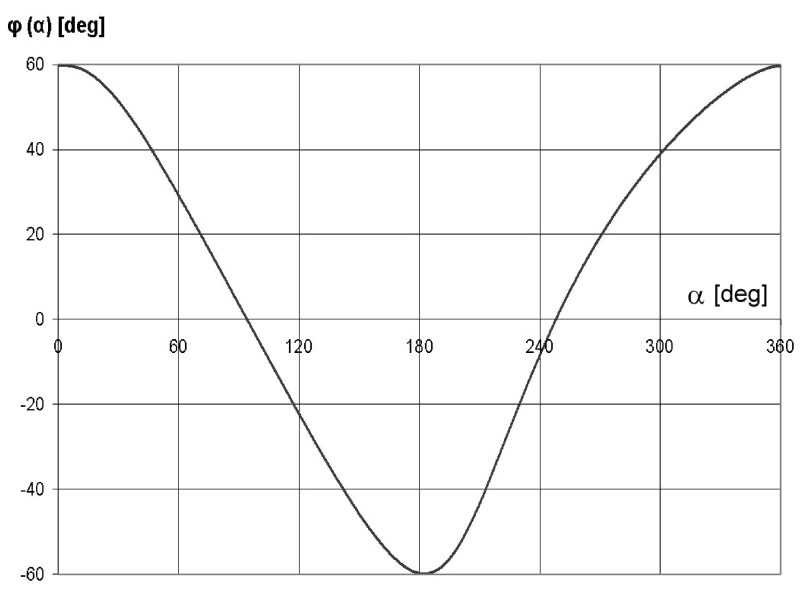

Fig. 5. Angular displacement $\varphi(\alpha)$ 
Oilless and frictionless compressor with double-sided rotationally oscillating piston

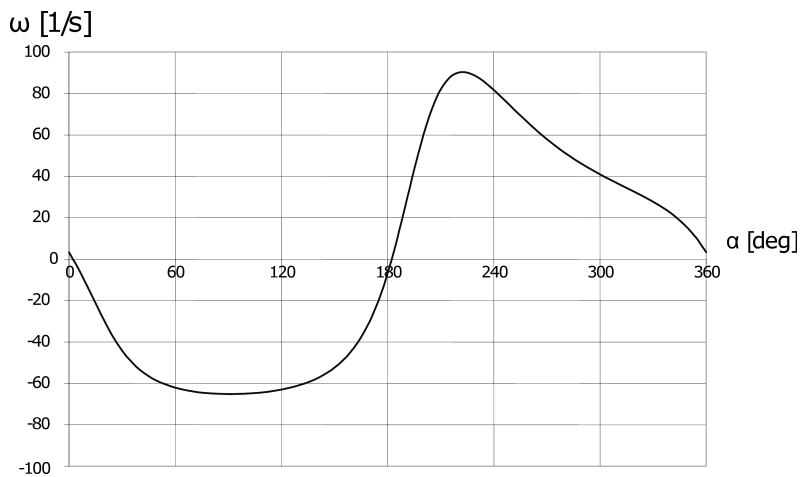

Fig. 6. Angular velocity $\omega(\alpha)$

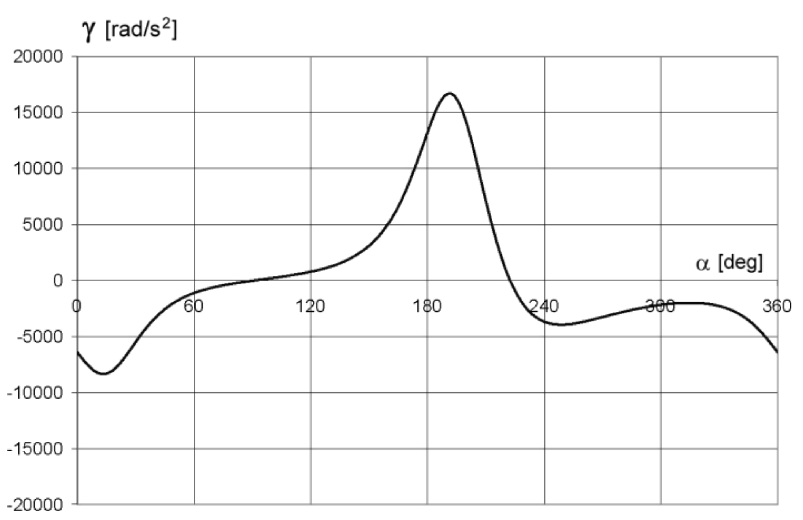

Fig. 7. Angular acceleration $\gamma(\alpha)$

Despite these irregularities, which may lead to some problems with balancing of the system, the simplicity of the structural solution of the drive system still is a great advantage.

Next, the loading of the drive system in complex operational conditions has been analysed. The pressure of the medium in the tank was assumed to be $P=1 \mathrm{MPa}$. The angular velocity was increased up to $n=900 \mathrm{rpm}$. The results are shown in Fig. 8.

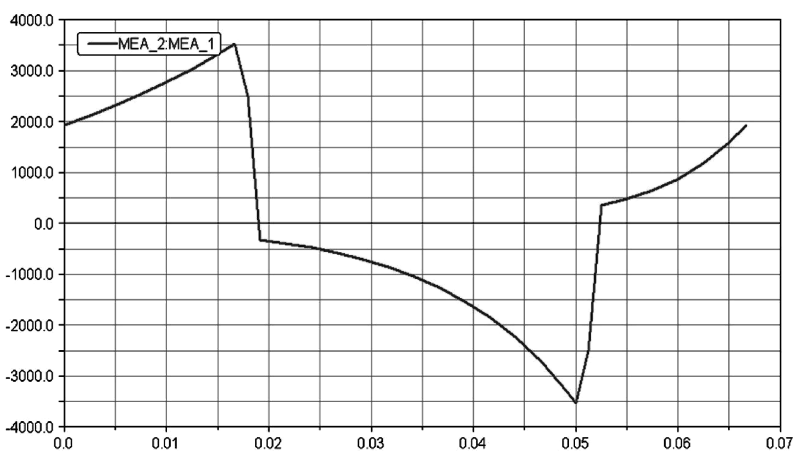

Fig. 8. Force acting on the vane for the full working cycle at compression pressure $1 \mathrm{MPa}$

Let analyse now the second variant of the drive system shown in Fig. 9.

As can be seen in Figs. 10-11, the angular displacements, velocity and acceleration are much more symmetrical in variant II. Particularly important is the course of acceleration as it enables inferring about loading of the drive system related to inertia of its elements.

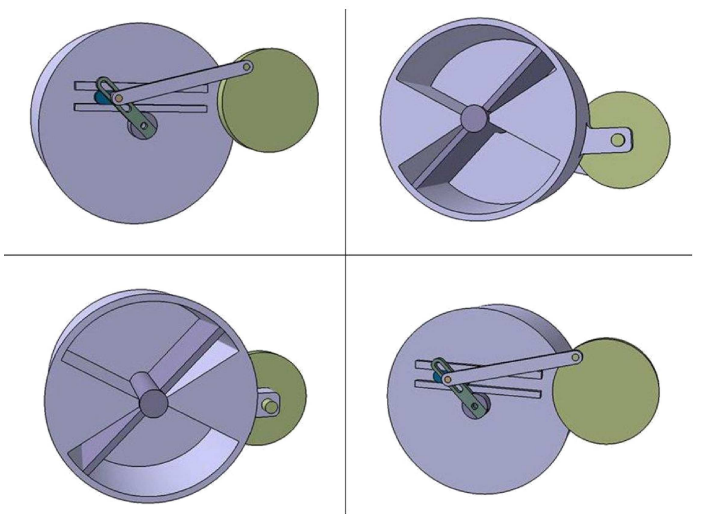

Fig. 9. Test model of the compressor drive system (variant II)

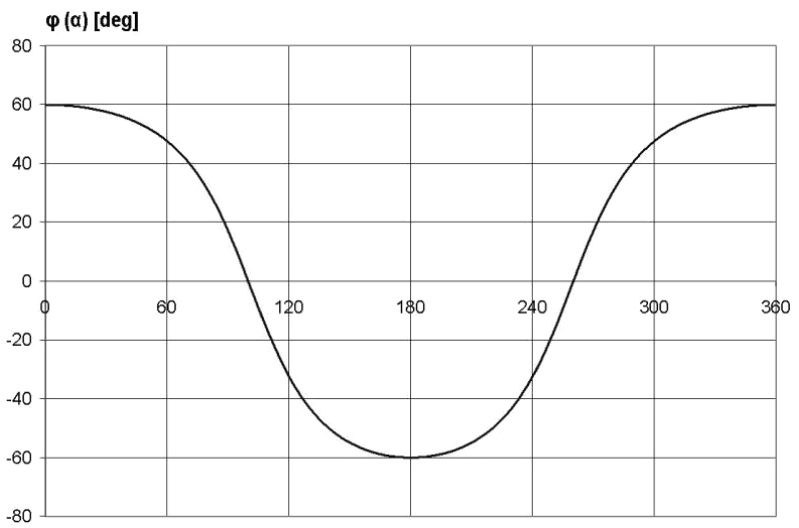

Fig. 10. Angular displacement $\varphi(\alpha)$ (variant II)

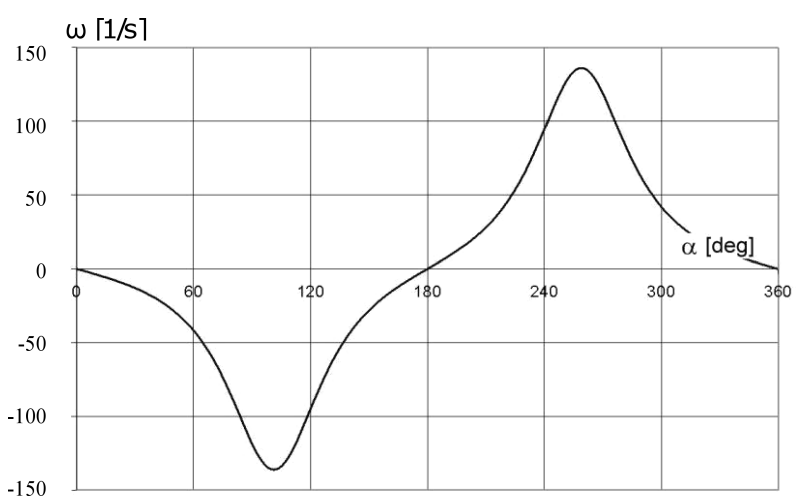

Fig. 11. Angular velocity $\omega(\alpha)$ (variant II)

Both, the angular velocity and acceleration corresponding to variant I and II are juxtaposed in Figs. 12-14.

The maximum instantaneous acceleration of variants I is roughly $10 \%$ greater than the top acceleration for variant II. Kinematically, the second solution is better than variant I, however its structure much more complicated. This is a big disadvantage because a larger number of cooperating parts increases energy losses due to friction (which is to be minimised by design) as well as additional sources of wear and an enlarged total backlash in the system. Another problem is higher cost of manufacturing. Finally, the simplicity of the construction made variant I more appropriate for the drive system of such a type of compressor. 
W. Chomczyk et al.

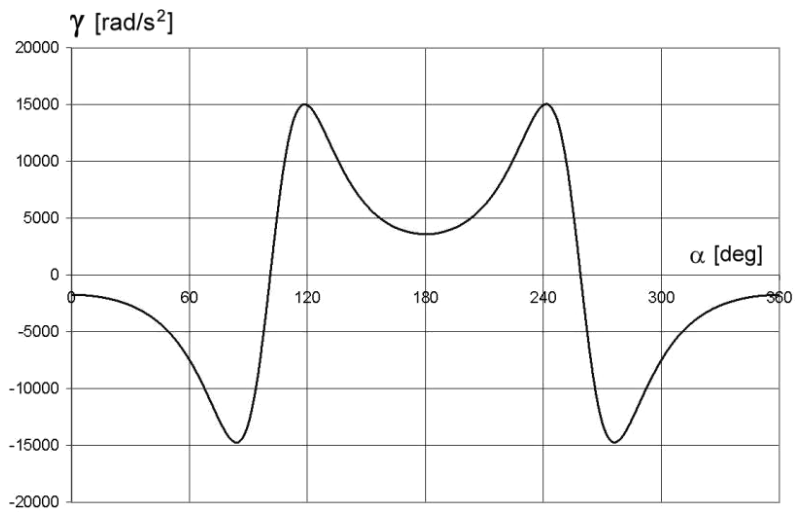

Fig. 12. Angular acceleration $\gamma(\alpha)$ (variant II) $\omega[1 / \mathrm{s}]$

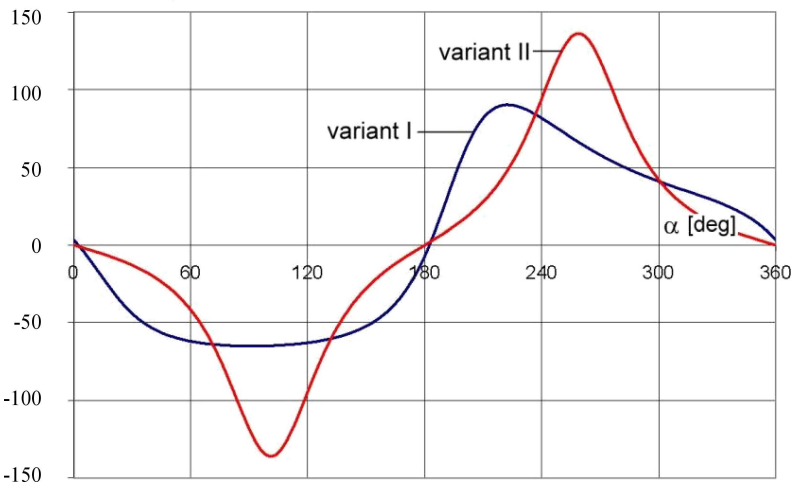

Fig. 13. Comparison of angular velocities for both variants

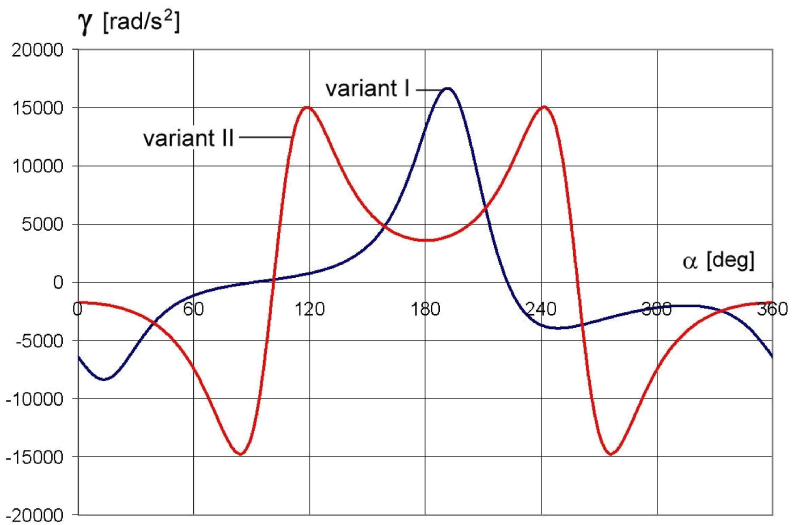

Fig. 14. Comparison of angular accelerations for both variants

A CAD model was created and thoroughly examined by numerical simulations. By changing the length of the connecting rod $L$, the effect of the ratio $L / R=\delta$ on the course of angular acceleration was investigated.

As can be seen in Fig. 15, growth of the ratio between the length of the connecting rod $L$ and length of the rocker $\operatorname{arm} R$ decreases the maximum positive accelerations and increases the maximum negative ones, i.e. equalizes both the absolute maximum values. The most disadvantageous is the acceleration course at $\delta \rightarrow \infty$. Obviously, the final dimensions $L$ and $R$ are an effect of a compromise between the compactness of the compressor and its strength and reliability, hence these crucial parameters will be set up at a further stage of designing.

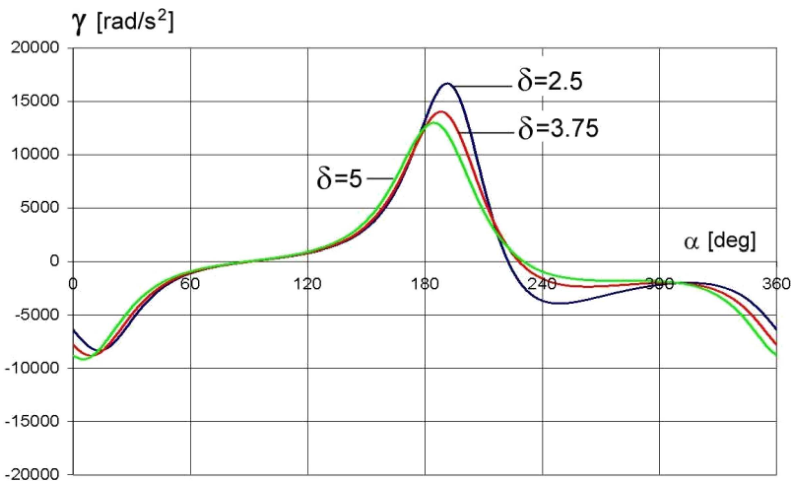

Fig. 15. Angular accelerations for different values of $\delta$

As far as the dynamics of the drive system is concerned, according to the model shown in Fig. 16 and the corresponding results of simulation (Figs. 16 and 17), it is seen that the inertia forces and pressure have different courses and opposite senses at their maxima. This enables lowering the resultant reaction on the crank-pin as well as on the compressor shaft through selection of the masses and geometry of the whole system.

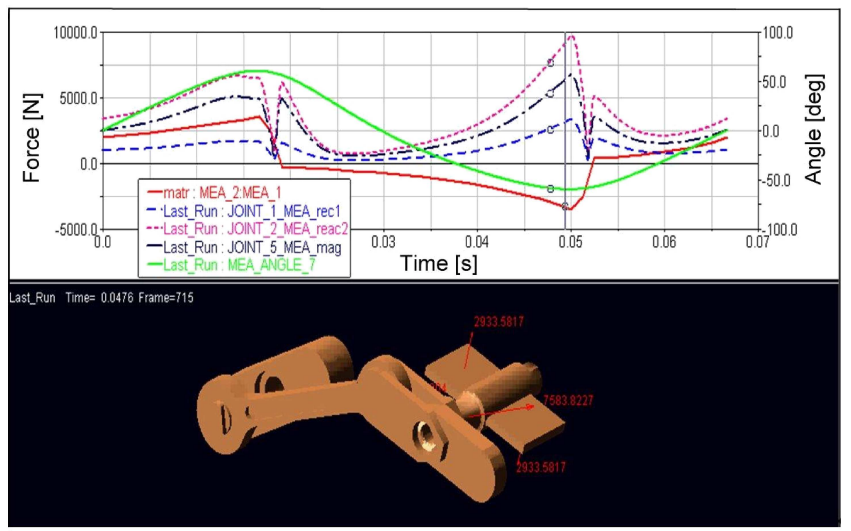

Fig. 16. Model of the drive system created in ADAMS. Example for full rotate of engine shaft in time 0.07 [s]

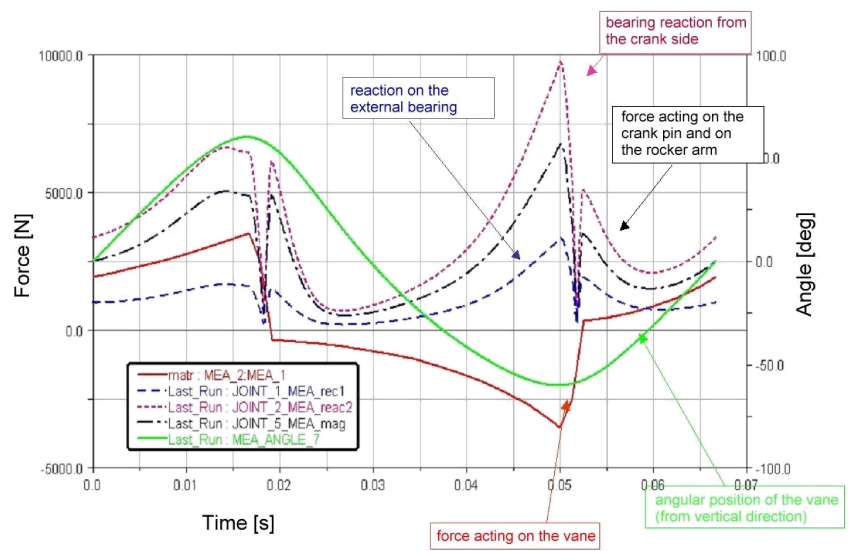

Fig. 17. Course of loadings $[\mathrm{N}]$ in the assumed drive system for given operational conditions

An advantageous distribution of loadings is related to the given operational conditions. Hence, the optimization should be made for the limit pressure of the medium in the tank 
(Fig. 6). The pressure in the cylinder chamber will be slightly higher.

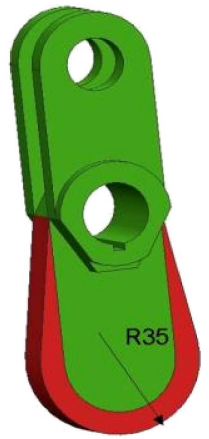

Fig. 18. Example of optimization of the crank shape in the drive system

The introduced modifications lowered the reaction on the vanes by $2000 \mathrm{~N}$ at the external position of the crank and enlarged by $1000 \mathrm{~N}$ at the internal position. There are, admittedly, two maxima of the loading acting on the crankshaft system (compare Figs. 17 and 19 after modification).

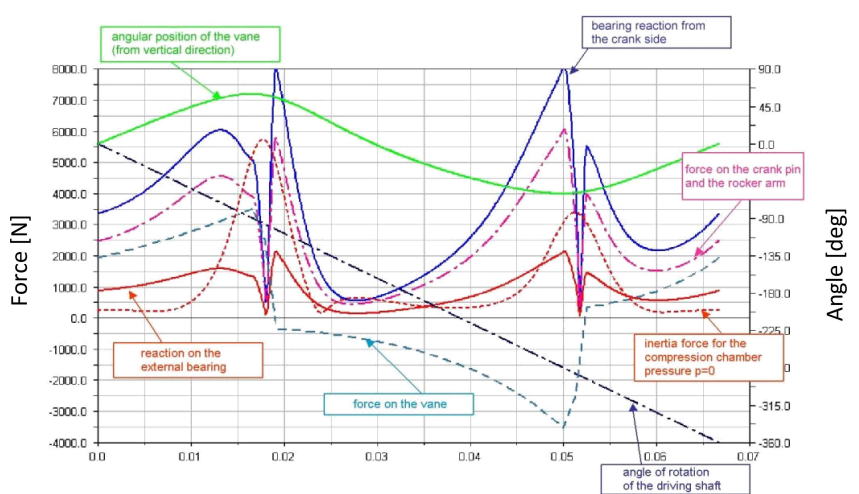

Fig. 19. Forces in the drive system after introducing optimization modifications

\section{Timing gear of the compressor}

The timing gear of a compressor is a system which sets the moment of opening of the valves, time of filling-in and pressing-out. The fundamental requirements for the timing gear are: leak tightness, possibly the greatest cross-section of the flow at the lowest circumference, small flow resistance (induced by the change of flow direction and its cross-section) low stroke and mass of movable parts of the valves allowing the drive system running really fast, assuring small clearance volume, quiet operation, high durability and low cost of manufacturing. A self-acting timing has been applied in the considered compressor: the opening and closing of the valves is controlled by a spring just after the pressure behind and in front of the valve is equalized. The drawback of such a solution is the necessity of keeping low stroke of the valves, which - for fast-speed compressors - forces the diameter of the valves to be great enough, which - in turn - enlarges the clearance volume. In the considered case of a bilateral, oilless and frictionless compressor, the velocity is reasonably low for application of a simple by design the self-acting timing gear. Poppet, plate and ring valves have been analysed. The poppet valves are characterised by a considerable inertia and complex construction, whereas the plate ones have big clearance volume which makes the flow slower. That is why the ring valve has been decided to be incorporated in the prototype of the compressor (Figs. 20, 21).

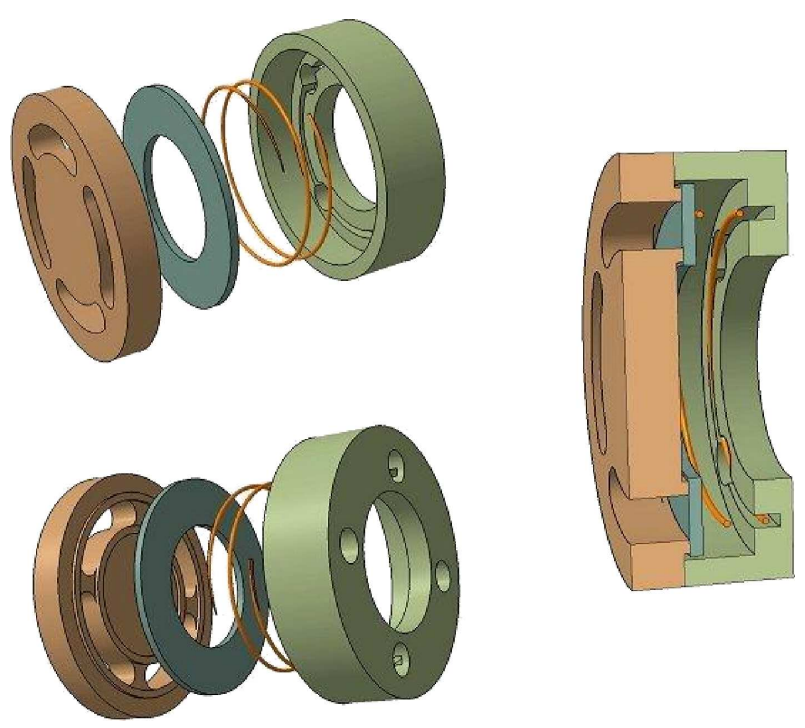

Fig. 20. CAD model of the ring valve with the simplified construction
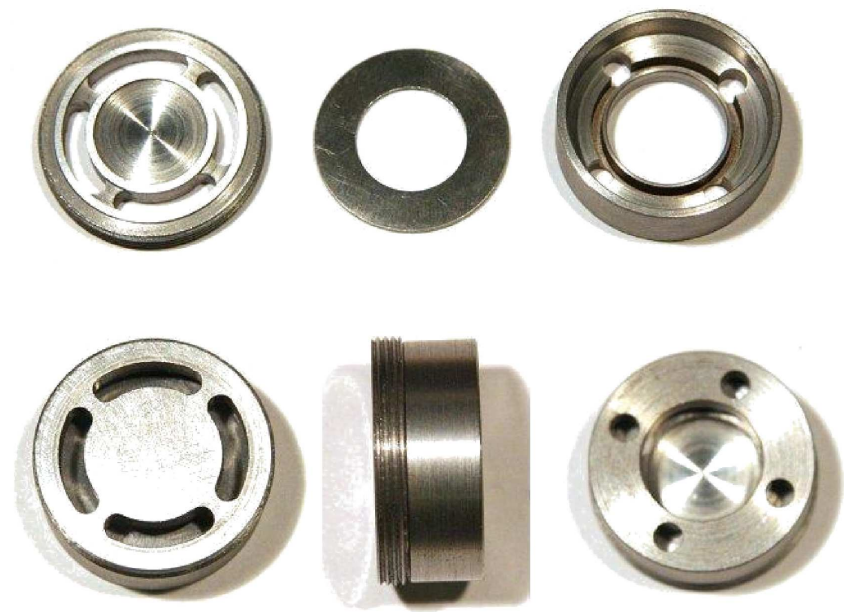

Fig. 21. Prototype of the ring valve worked out for the oscillatory compressor

An own design has been worked out and optimised in terms of the compressor efficiency. The limitation was the overall dimension. The maximum allowable diameter of the valves placed in the baffles, two for each pressure chamber, is $32 \mathrm{~mm}$. A parametrised CAD model of the valve has been prepared. Dimensions of the valve have been initially assumed, and then correlated. After that, a relationship for the first and second cross-section area $P_{1}, P_{2}$ in function of $d$ and $D$ has been derived, see Fig. 22. 
W. Chomczyk et al.

\begin{tabular}{|l|l|}
\hline Stop & Ring \\
variable dimension: & fixed dimensions: \\
$d_{1}=16.7 \mathrm{~mm}$ & $z_{1}=0.25 \mathrm{~mm}$ \\
fixed dimensions: & $z_{4}=0.5 \mathrm{~mm}$ \\
$D_{\max }=32 \mathrm{~mm}$ & $h_{p}=1 \mathrm{~mm}$ \\
$z=2 \mathrm{~mm}$ & resulting dimensions: \\
$d_{r}=20 \mathrm{~mm}$ & $D_{p}=D_{1}-2 z_{1}$ \\
$h_{r}=1.5 \mathrm{~mm}$ & $D_{p}=27.5 \mathrm{~mm}$ \\
$l_{r}=1 \mathrm{~mm}$ & $d_{p}=d_{1}-2 z_{4}$ \\
$l_{l}=3 \mathrm{~mm}$ & $d_{p}=15.7 \mathrm{~mm}$ \\
resulting dimensions: & \\
$D_{1}=D_{\max }-2 z=28 \mathrm{~mm}$ & \\
\hline
\end{tabular}

$$
\begin{aligned}
& \text { Area of the first cross-section (P1) } \\
& a_{1}=\frac{D-d}{2} \quad a_{1}=3.4 \mathrm{~mm} \\
& a_{1}=\arcsin \left(\frac{\frac{a_{1}}{2}+b_{1}}{\left.\frac{D+d}{4}\right) \quad \alpha_{1}=14.478 \mathrm{deg}}\right. \\
& P_{1}=\left(\frac{\pi D^{2}}{4}-\frac{\pi d^{2}}{4}\right) \frac{360^{\circ}-8 \alpha_{1}}{360^{\circ}}+\frac{4 \pi a_{1}^{2}}{4} \\
& P_{1}=192.808 \mathrm{~mm}^{2}
\end{aligned}
$$

Seat
fixed dimensions:
$l=4 \mathrm{~mm}$
$z_{2}=1.25 \mathrm{~mm}$
$z_{3}=1.25 \mathrm{~mm}$
$b_{1}=1 \mathrm{~mm}$
$c_{1}=0.5 \mathrm{~mm}$
resulting dimensions:
$d=d_{p}+2 z_{3}$
$d=18.2 \mathrm{~mm}$
$D=D_{p}-2 z_{2}$
$D=25 \mathrm{~mm}$

\section{Area of the second} cross-section (P2)

$$
\begin{aligned}
& P_{2}=\pi\left(\frac{d_{p}}{2}\right)^{2} \\
& P_{2}=193.593 \mathrm{~mm}^{2}
\end{aligned}
$$

Fig. 22. Calculations of cross-sections $\mathrm{P}_{1}$ and $\mathrm{P}_{2}$ made in MathCad

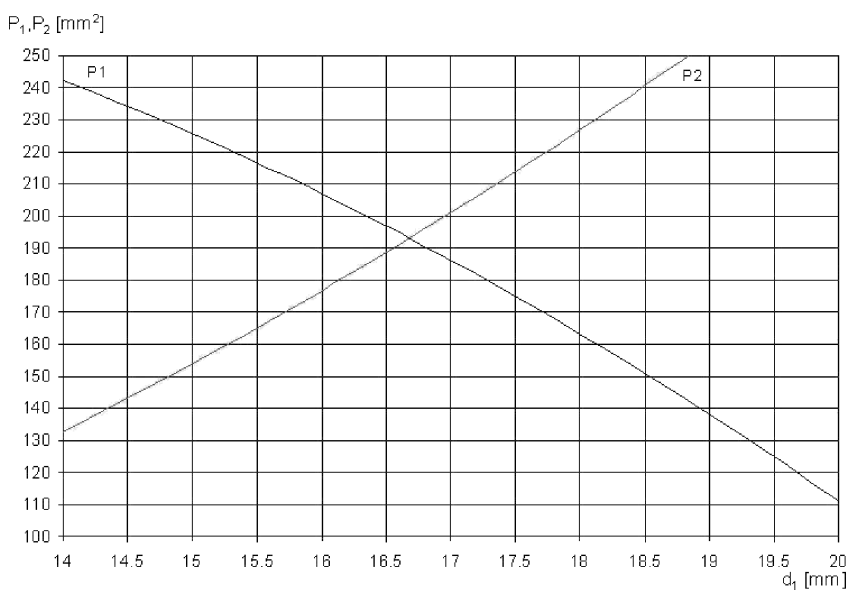

Fig. 23. Optimization diagram for the ring valve with the simplified construction

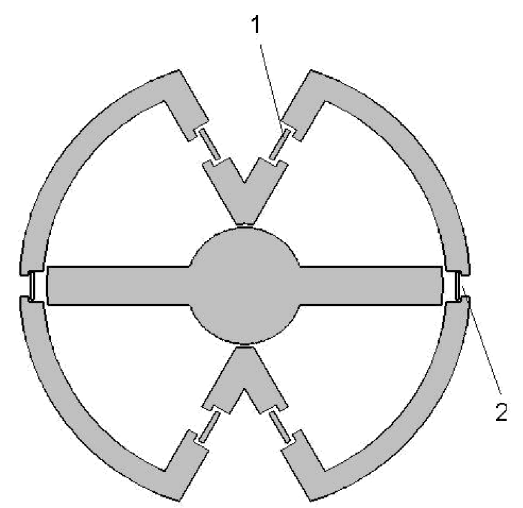

Fig. 24. Placement of valves: 1 - outlet valve, 2 - inlet valve

Most piston compressors are equipped with valves mounted in the head, as it is an optimal solution. As far as oscillatory compressors are concerned, the problem is much more complicated. The place corresponding to the head of reciprocating compressors is the baffle (partition wall) in oscillatory compressors. Because of a specific construction of such devices, the space free for mounting the valves in the baffles is not big indeed. This fact inclines the designer to look for other solutions for placing the valve in the compressor. Below, the considered variant is discussed - the pressure valve mounted in the baffles, the suction ones in the chamber. Such a constructional solution is depicted in Fig. 35. It does however, affect the course of indication. A considerable drawback here, is an impulsive character of operation of the suction valves. Sudden opening of the valves brought about by high underpressure generates noise and vibration. Additionally, the possibility of supersonic gas flow is also an undesirable effect. The main disadvantage of such a solution is the lack of problems with space for valves, which allows the designer to apply any kind of valves appropriately selected for the oscillatory compressor.
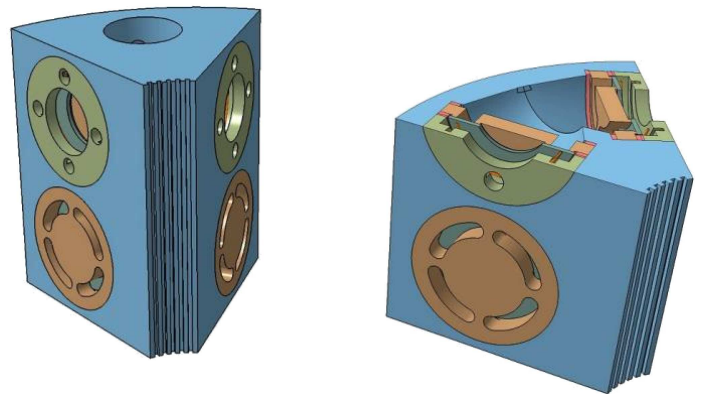

Fig. 25. Baffle of the oscillatory compressor with built-in ring type valves

\section{Pressure chamber}

The pressure chamber in the oscillatory compressor is comprised of several elements. The main dimensions and parts belonging to it are shown in Fig. 26.

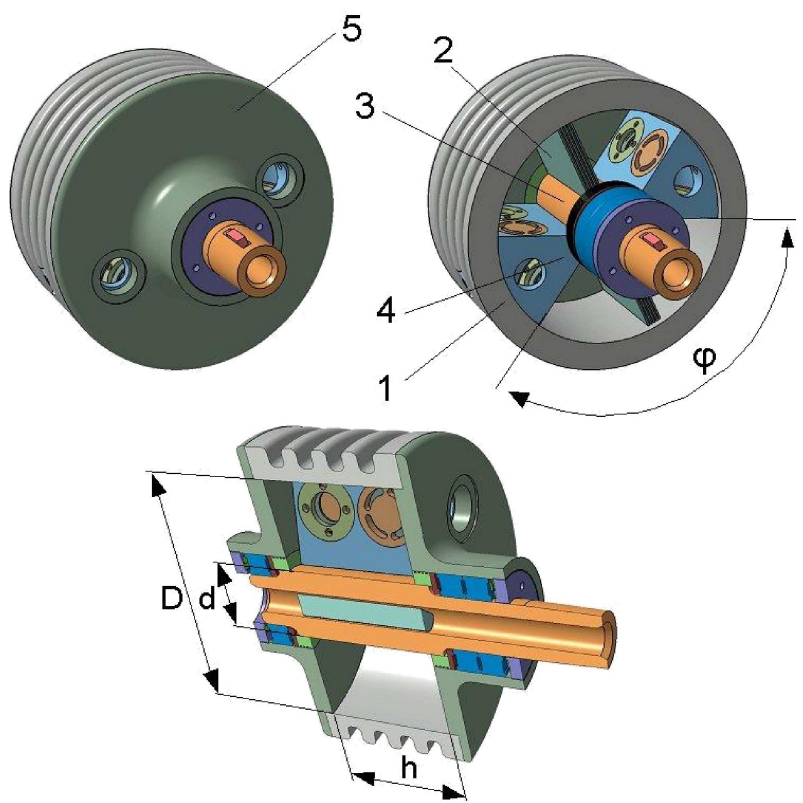

Fig. 26. Pressure chamber of the oscillatory compressor: $1-$ cylinder, 2 - vane, 3 - shaft, 4 - baffle, 5 - cover 
The diameter $D$ and height $h$ as well as the maximum working angle $\varphi$ are the characteristic dimensions for such a type of compressor, and have been initially set up at the beginning of designing the entire mechanism. Other dimensions, such as vane thickness and shaft diameter $d$ result from strength analysis. It has been initially assumed that the lower limit of rotation speed would not drop below $n=750 \mathrm{rpm}$, and the nominal one would be established during experimental tests on a real prototype, and - expectedly - would depend on operational efficiency of the valves.

\section{Structural design of the vaned shaft}

Technological reasons, more precisely - high demand for quality of the cylindrical surface of the pressure chamber responsible for tightness between the shaft and baffles, excluded in practice the possibility of making use of the shaft and vanes as a single elements manufactured, e.g. by forging. Contactless, labyrinth seal requires precisely distributed gap between the elements, kept within $0.02-0.04 \mathrm{~mm}$. That is why the outer surface of the shaft must be finished by grinding before the assembling (Fig. 27b). Another problem to be solved was the way of connecting the shaft with vanes. Among many proposals, like welding or studding, two most promising methods have been selected. The first one that attracted particular attention was joining the elements through the so-called dovetail (see Fig. 27a).

a)

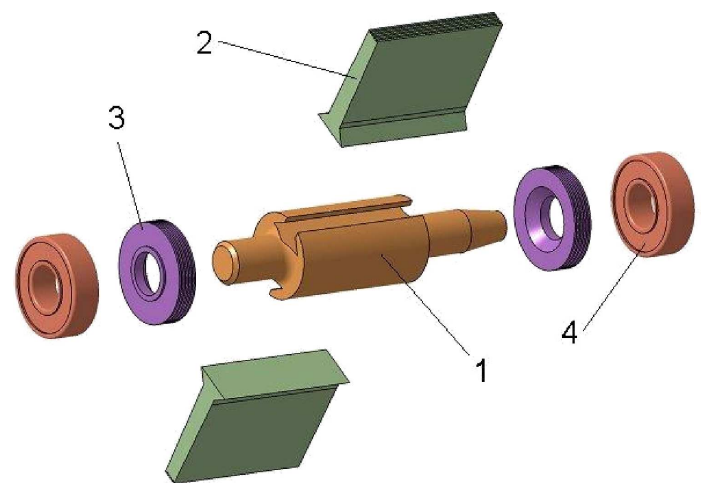

b)

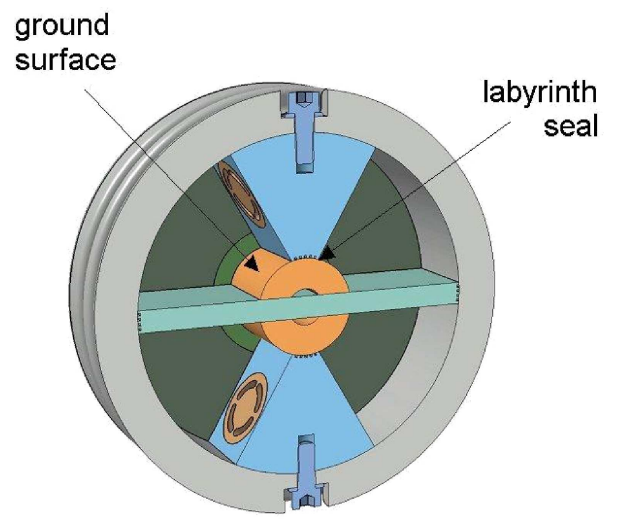

Fig. 27. a) Connection between the shaft and vane through a dovetail: 1 - shaft, 2 - vane (piston), 3 - ring seal, 4 - bearing; b) Seal between the shaft and baffles a)

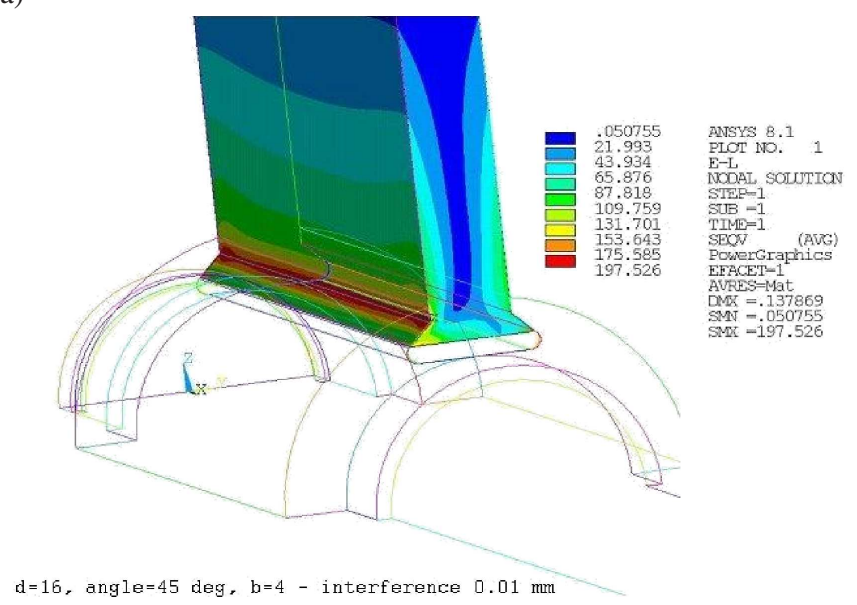

b)

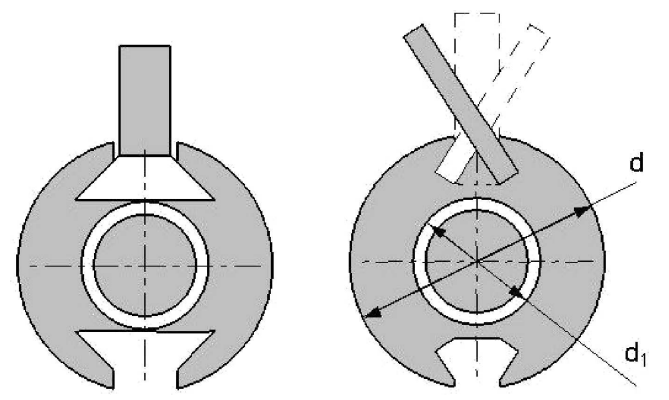

Fig. 28. a) Reduced stresses in the vane (ANSYS); b) milling of the socket: with a profile cutter (left), with a plain milling cutter (right)

Unfortunately, such a connection is far from ideal. There appeared many technological problems with manufacturing the socket for the vane. One of the possibilities was to mill the socket with a profile cutter (Fig. 28a). Another one was to make use of a plain milling cutter (Fig. 28b). The biggest problem, however, was the question of strength. Investigations harnessing the finite element method through application of ANSYS software revealed that the stresses generated by the maximum pressure acting on the vanes $\left(P_{\max }=1 \mathrm{MPa}\right)$ reach locally roughly $200 \mathrm{MPa}$ (Fig. 28a), which is far too big even for materials of highest quality. Obviously, another solution to the issue was necessary to be found. Finally, it was decided to employ a forced-in joint between the shaft and vanes (Fig. 29). For the needs of the prototype, the blade groove was manufactured by milling. In a series production, such a method would be too time consuming. In the process of destination, the shaft might be pre-treated by forging, and then the groove finished by broaching. The assembly would then require a special holder to ensure appropriate mutual setting of the parts top be joined. The final surface machining of the vanes, i.e. grinding to ensure sufficient tightness of the compressor (between the vane and chamber as well as the vane and baffles) would be done after assembling the shaft with the vanes.

The stress state for the forced-in joint was calculated by FEM using ANSYS, and is shown in Fig. 29b. The resulting stresses are much less than in the dovetail case - their maximum did not exceed $120 \mathrm{MPa}$. 
a)
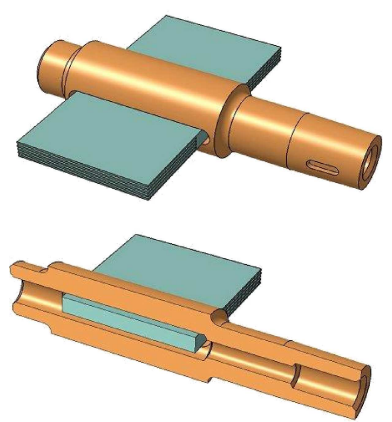

b)

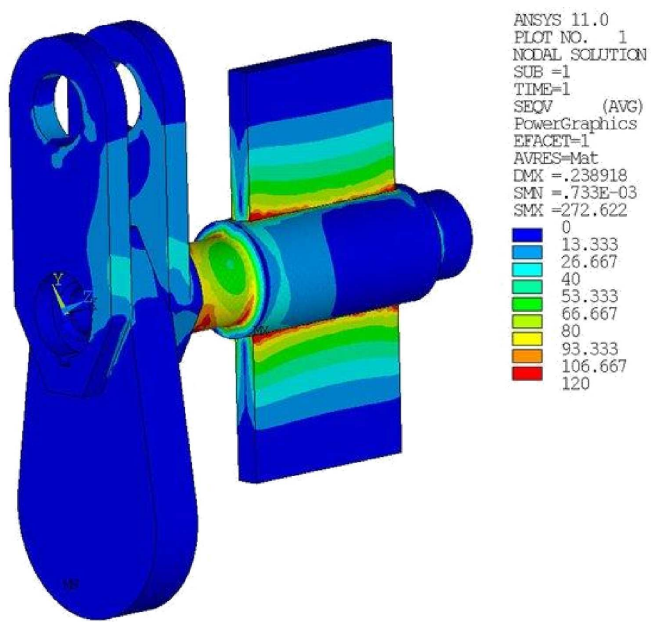

Fig. 29. a) View to the cross-section of the shaft and the forced-in vane; b) reduced Huber-Misses stresses

\section{Manufacturing of the prototype compressor}

On the basis of carried out simulations and analyses, an initial technical documentation of the prototype oilless and frictionless compressor with a rotary oscillating piston has been worked out. Because of the peculiarity of the construction, the following problem required to be overcome and solved: manufacturing technology of the bladed piston (vanes), ensuring sufficient accuracy of the machining of the crucial surfaces (chamber, vanes, baffles, cover) in terms of the assumed symmetry and gap distribution parameters $(0.02 \div 0.03) \mathrm{mm}$.

To achieve this, non-uniform distribution of the working temperature and the resulting deformations affecting the value of gap had to be taken into account. The prototype case (the cylinder with the cover) was decided to be made traditionally of grey cast iron. The shaft vanes of an alloy steel as separate parts to be joined by forcing the vanes in the grooved shaft. The partition walls (baffles) should also be made of an alloy steel. Similar coefficients of thermal expansion of the cast iron and steel are expected to maintain the gap within assumed limits in operating conditions. Another issue were the valves. That was confirmed during experiments. Three questions can be underlined here: the problem of leakage, drop of efficiency due to flow chocking at higher rotational speeds, and the problem of inertia loading at greater working frequency (too big mass of moving elements and out-of-phase operation of the valves). The authors decided to apply com- mercial ring valves being used in Polish compressors 3JW60. Alternately, valves of own design had been worked out as well. After initial verification of the prototype at the end of experimental investigations, suction valves for pneumatic systems were incorporated and mounted in the cover. In the baffle, on the other hand, in the place of the suction valve, an additional pressure valve was built-in. At the final stage of modifications introduced to the prototype, there were two pressure and two suction valves in each chamber of the compressor.

a)

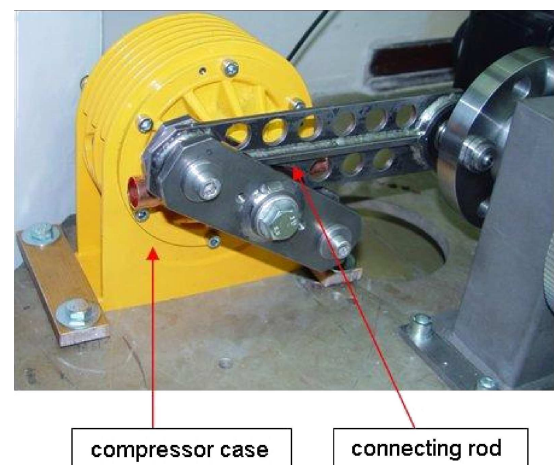

b)

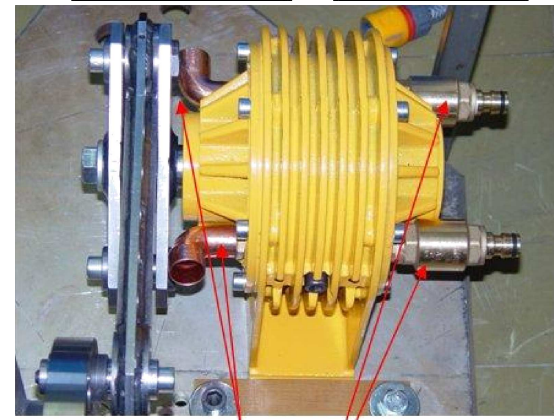

c)

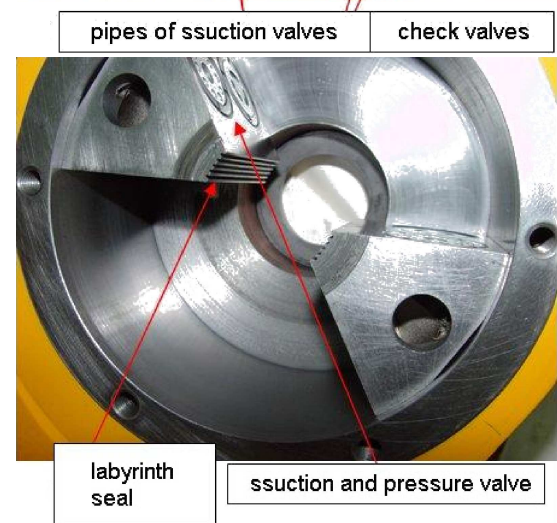

d)

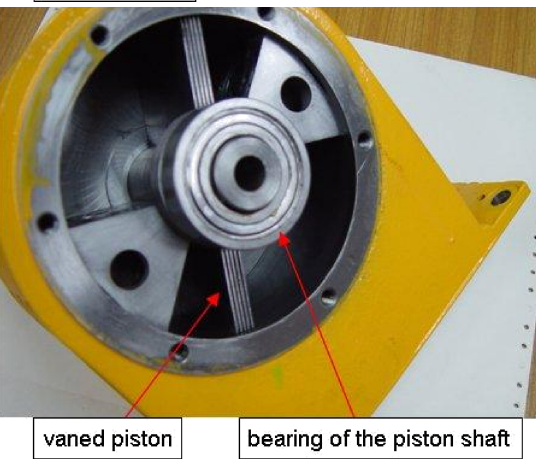

Fig. 30. Physical model of the oilless compressor with a double-sided piston - assembled and in parts 
During tests at moderate and high pressure of the medium, there appeared a problem of momentary loss of the gap and the occurrence of frictional contact between the vane and the cover (Fig. 33). This was brought about by a non-uniform thermal loading and mechanical loading from the crankshaft system (the unbalanced radial component of the dynamic forces). The problem was solved by introducing an intermediate support in the drive system and fan cooling.

In Fig. 30, the elements and the whole assembly of the considered prototype with pressure and suction valves placed in the baffles (Fig. 30d) are shown. The crankshaft system driving the compressor without the intermediate support is depicted in Figs. 30a,b. Suction pipes and reduction valves in the pressure ducts are shown in Fig. 30b. The labyrinth seal of the bladed piston and the baffles is illustrated in Figs. 30c,d.

The final version of the prototype after verification assumes introduction of additional pressure valves in the place of suction ones in the baffle, and mounting the suction valves in the cover - see Sec. 9, Fig. 34.

\section{Elaboration and manufacturing of the test stand for experimental investigation}

Into the composition of the test stand, the following elements and assemblies entered:

- power source - Lenz's electric motor with a controller enabling continuous regulation of the rotation speed within 0-1450 rpm and a torque-meter,

- transmission system reducing the speed,

- crankshaft system,

- the object of study - oilless, frictionless compressor,

- pressure tank for the compressed medium having volume of $100 \mathrm{dcm}^{3}$ and being equipped with pipes, valves and a pressure pick-up,

- measuring apparatus - pressures gauges, thermometers, angular position sensors, flow-meter, computer with ESAM CF software for data registration,

- vibroinsulating plate with fixing elements.

The test stand enabled investigation of the compressor for rotation speeds within $n=0-1000 \mathrm{rpm}$ after reduction, or up to $1450 \mathrm{rpm}$ without it. The engine (electric motor) with a surplus power and torque allowed examination of compressors with higher efficiency. The certificated tank stored $100 \mathrm{dcm}^{3}$ of the medium (air) compressed up to 10 bar. For measurement of pressure in the chambers and baffles, commercial gauges Johnson Controls P499VBS-401C were used.

The employed software enabled digital registration and then visualization of the data gathered by the pressure gauge, angular position sensor and the flow-meter in function of time or angular displacement (volume after re-scaling). This allowed the investigators to currently draw the indicator diagrams. Temperature measurements were realised with help of thermocouples and a thermovision camera.
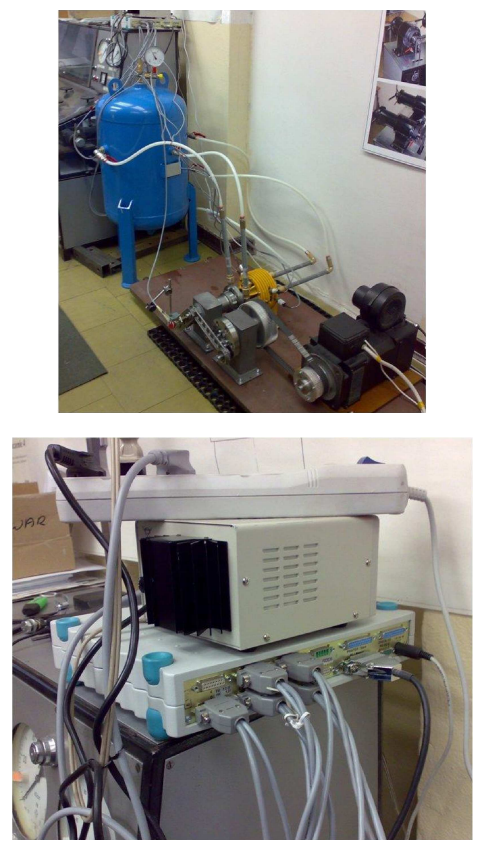

Fig. 31. Test stand with the measuring apparatus

\section{Qualitative studies on the prototype}

Experimental investigations of the prototype compressor were carried out on the test stand described in the previous Section. Tests checking the quality of assembling and operating were done for small rotation speeds $n=0-250 \mathrm{rpm}$. Next, after controlling the setting and fixing of the compressor, the air tank was connected, which was followed by pressure measurement with a dial gauge. At the crankshaft rotation speed $n=450 \mathrm{rpm}$, the pressure reached 4.5 bar in 4 minutes. Further tests were realised after installing pressure gauges in the chambers and baffles of the compressor. That allowed the assessment of the operation of the valves. Achievement of 6 bars in the tank did not make any problem. However, the pressure in the chambers was too big comparing to that in the tank (see Fig. 35). Also the temperature distribution in the outlet pipes was very non-uniform - Fig. 32. This bespoke an excessive chocking of the forced-out air on the valves as well as leakage in the chambers at the left side.

The drop of pressure in the left outlet pipe was eliminated by milling the seat having threaded mounting of the check valve and by eliminating its skeweness. The non-uniformity of temperature distribution left, however less severe.

The test were continued for increasing rotation speeds up to $n=0-1000 \mathrm{rpm}$. Due to pressure asymmetry in the left and right chamber, which appeared for higher pressures, to conical pivot of the piston shaft turned with relative to the hub of the rocker arm (elasto-frictional joint), which consequently led to the pressing of the vanes against the baffle at the pressure side. Traces of rubbing are shown in Fig. 33. Rubbing also occurred on the middle part of the shaft in the contact region with the labyrinth seal. This was - as precisely measured after disassembling the prototype - due to deformations brought about by temperature growth in the thermo-compressed bonding between the shaft and vanes. 
W. Chomczyk et al.
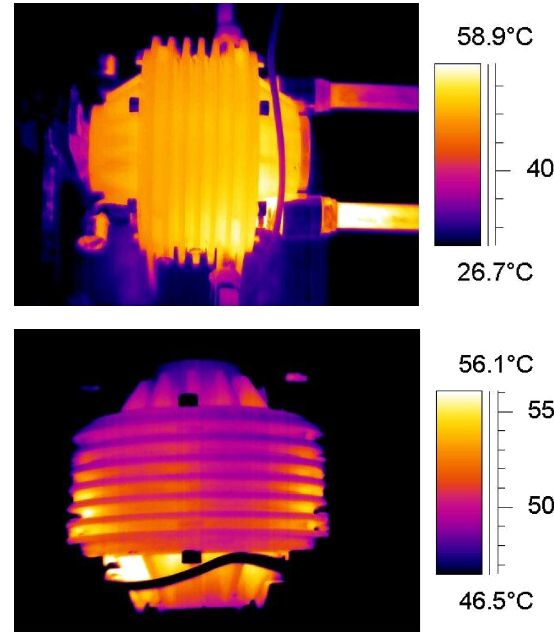

$56.1^{\circ} \mathrm{C}$

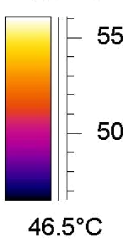

Fig. 32. Temperature distribution in the prototype compressor under low loading conditions $\left(\mathrm{P}_{\text {tank }}=0.6 \mathrm{MPa}\right)$
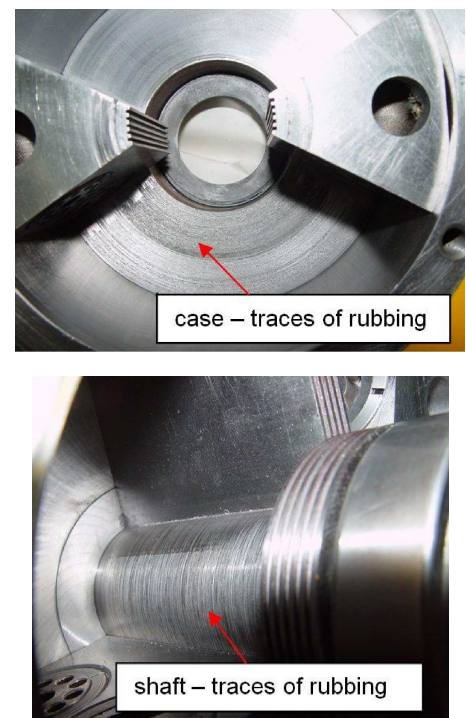

Fig. 33. Visualization of the rubbing traces
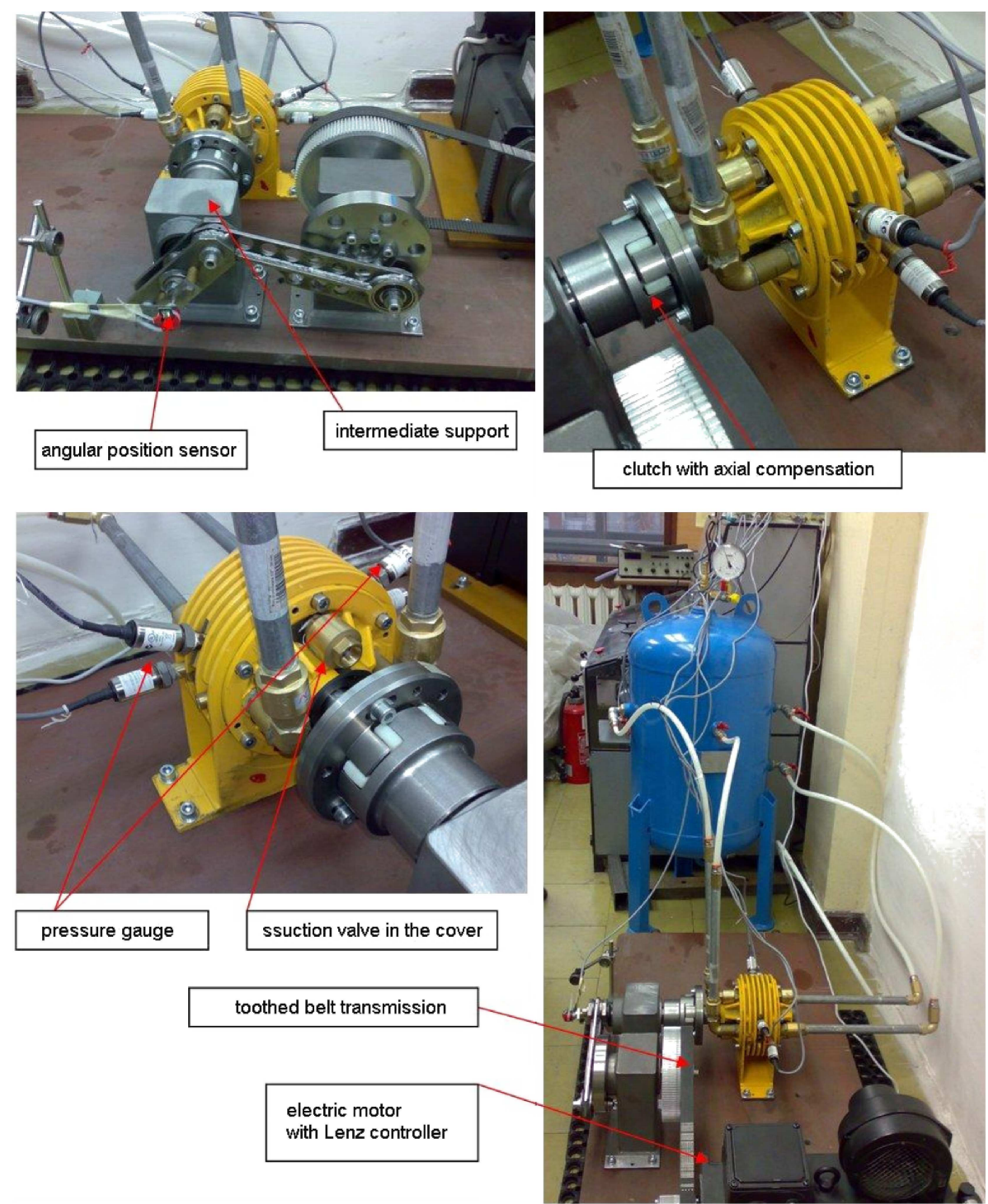

Fig. 34. Test stand with the modified compressor fully furnished with measuring apparatus 
Oilless and frictionless compressor with double-sided rotationally oscillating piston

Grinding-in with a specially designed device partly eliminated the asymmetry. At higher speeds, the unbalanced radial components of dynamical forces bent the shaft and skewed it within limits of the radial gap of the rolling bearings and their fit in the cover seats. This generated an axial component of the dynamic force which shifted the vaned shaft towards "pressure" baffle. The total clearance amounted $0.025 \mathrm{~mm}$. Its distribution was manually established while assembling the system. At those values, the symmetrical distribution during operation was unachievable. Consistently, it was decided to unload the vanes by making use of an intermediate support taking the direct load from the connecting rod and transferring pure torque to the vaned shaft - Fig. 34. Following tests on a modified drive system and dimensional correction of the shaft revealed better operation of the compressor. There was no rubbing observed, and the generated pressure in the tank reached 8 bars - Figs. 35 and 36. The results were better, however still not fully satisfactory. The working pressure in the chambers was too still big, and the compressor excessively heated up. Too low flow capacity of the valves did not allow developing full efficiency of the compressor. In the given constructional and technological conditions, an additional pressure valve in each baffle was installed in the place of the suction valve, which - in turn - was moved to the only left area - the cover. Check valves with a considerable crosssection were used for the suction valves. Unfortunately, the are characterised by an enlarged inertia. After that modification, the compressor generated $5.5 \div 6.0$ bars in the tank in $3.5 \mathrm{~min}$. at barely $n=220 \mathrm{rpm}$. Yet a higher pressure was unachievable despite augmenting the speed up to $n=450 \mathrm{rpm}$. The temperature rapidly grew, but the pressure was increasing very slowly. The reason was too big inertia of the suction valves. It was decided then to replace these valves once again, this time with valves having a smaller cross-section and inertia, resembling the construction of the pressure valves. It was not an optimal solution, but facing the lack of alternative, it improved the working efficiency for higher rotation speeds. The pressure in the tank grew up to $8.0 \div 8.5$ bars at $n=850 \mathrm{rpm}$. No rubbing occurred, and the temperature in the left and right chamber became more evenly distributed. Still, the theoretical efficiency of the compressor was not gained. The suction valves did not secure sufficient input. Admittedly, there was no possibility of improving the problem essentially because of constructional issues that excluded the placement of bigger suction valves or making them double.
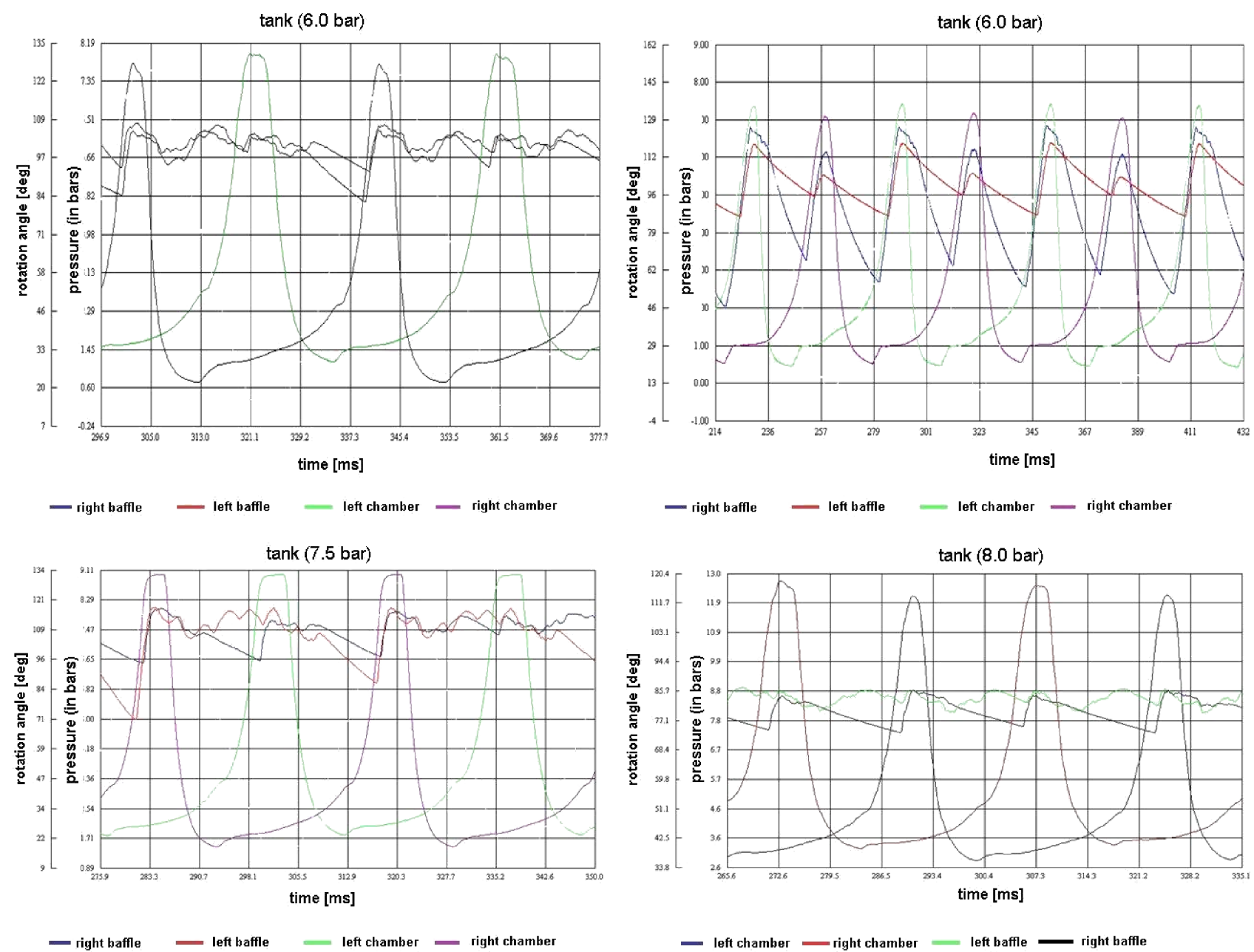

— right baffle $\quad$ left baffle

Fig. 35. Courses of pressure in both chambers (colour on-line) 
W. Chomczyk et al.
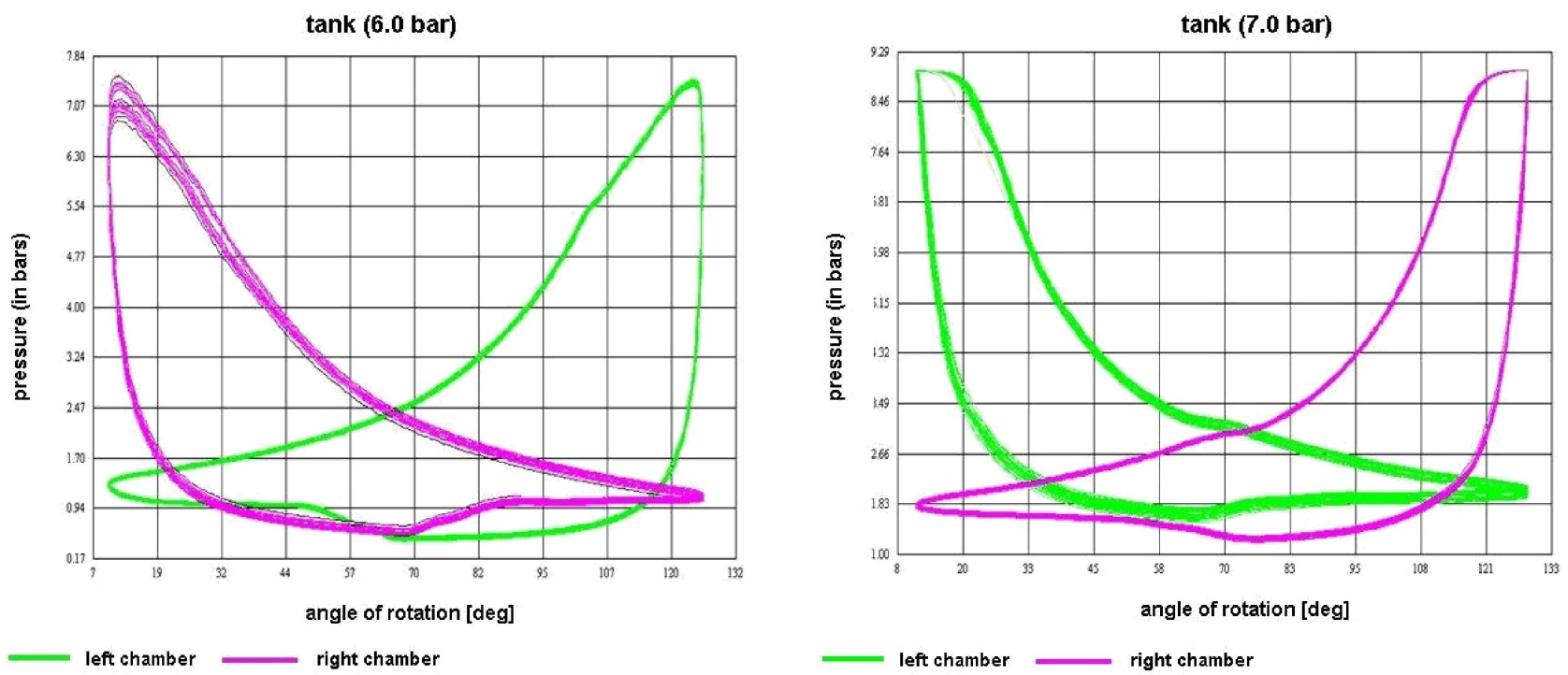

Fig. 36. Indicator diagrams based on pressure variations in both chambers according to measurements carried out on the test stand shown in Fig. 34

\section{Concluding remarks at the current stage of investigations}

- The fundamental task is the correct selection of valves the pressure ones should have greater capacity than that calculated theoretically on the basis of the chamber volume.

- The suction valves should have at least the same capacity as the pressure ones.

- The compressor design should compensate the effect of unbalanced dynamical forces.

- An optimal solution seems to be the compressor with a rotating piston and cylinder (patent P385734) allowing reduction of mechanical loading and ensuring better filling-in by giving more space for placing the pressure and suction valves.

- Introducing a thermo-compressionless joint for the vanes.

- Making use of cer-metals to lower the mass by half and to decrease the inertia loading.

- Consideration of an alternative design with the pressure valves mounted in the vanes and the suction ones placed in the baffles together with self-lubricating seals instead of the labyrinth ones.

- Optimization of geometrical parameters and stroke of developed digital model and the analytical relationship of the proposed suction valve, made it possible to increase the capacity of the ring valve.

- It should be noted that in the case of displacement machines with reciprocating piston move, minimize dynamic forces occurs only in a narrow range of speed and pressure on the blades of the piston.
- The forces of inertia of rod drive system and force the of the pressure on the piston have their maxima of opposite sign. They are closer to each other in absolute value, the resultant load is less.

- The worked out prototype confirmed the potentiality of achieving the assumed operational parameters after introducing the above discussed modifications.

\section{REFERENCES}

[1] W. Chomczyk, Multi-chamber Sucking-forcing-out Working Machine, Bialystok University of Technology, Białystok, (to be published), (in Polish).

[2] V. Chlumsky, Piston compressors, PWT, Warszawa, 1961, (in Polish).

[3] W. Chomczyk, Multi-chamber Working Machine with a Vaned Piston, Especially as a Compressor, Pump, Engine, Patent No. 68220, 1973, (in Polish).

[4] W. Chomczyk, W. Ostapski, and J. Piotrowski, Frictionless and Oilless Compressor with a Rotating Piston and Cylinder, Patent No. P385734, 2008, (in Polish).

[5] W. Chomczyk, W. Ostapski, and J. Piotrowski, Frictionless and Oilless Compressor with a Fixed Piston and Rotating Cylinder, Patent No. P385733, 2008, (in Polish).

[6] W. Ostapski and J. Piotrowski, Construction of an Unloaded Piston for Uplift Machines, Patent No. P394072, 2011, (in Polish).

[7] M. Hać, J. Lazęcki, and W. Ostapski, "Modeling of driving mechanism of oscillatory air-compressor", Machine Dynamics Problems 33 (3), 13-24 (2009).

[8] T. Tataj, "A design of an oscillatory compressor", MSc Thesis, Warsaw University of Technology, Warsaw, 2007, (in Polish). 